\title{
INTEGRAL REPRESENTATION OF THE DRAZIN INVERSE*
}

\author{
N. CASTRO GONZÁLEZ ${ }^{\dagger}$, J. J. KOLIHA ${ }^{\ddagger}$, AND YIMIN WEI ${ }^{\S}$
}

Abstract. In this note we present an integral representation for the Drazin inverse $A^{\mathrm{D}}$ of a complex square matrix $A$. This representation does not require any restriction on its eigenvalues.

Key words. Drazin inverse, Integral representation.

AMS subject classifications. 15A09, 65F20

1. Introduction. It is a well-known fact that if the eigenvalues of $A \in \mathbb{C}^{n \times n}$ lie in the open right halfplane, then the inverse of $A$ can be represented by

$$
A^{-1}=\int_{0}^{\infty} \exp (-t A) d t
$$

This representation was extended to the Drazin inverse by Koliha and Straškraba [2, Theorem 6.3] in the form

$$
A^{\mathrm{D}}=\int_{0}^{\infty} \exp (-t A)\left(I-A^{\pi}\right) d t
$$

for those singular matrices whose nonzero eigenvalues lie in the open right halfplane and for which $\operatorname{ind}(A)=1$; here $A^{\pi}$ is the eigenprojection of $A$ corresponding to the eigenvalue 0 . Recall that $\operatorname{ind}(A)$, the index of $A$, is the least nonnegative $k$ for which the nullspace of $A^{k}$ coincides with the nullspace of $A^{k+1}$.

Recently, Castro, Koliha and Wei [1, Corollary 2.5] obtained a simple integral representation of the Drazin inverse $A^{\mathrm{D}}$ for matrices $A \in \mathbb{C}^{n \times n}$ (and more generally elements of a Banach algebra) for which the nonzero eigenvalues of $A^{m+1}$ lie in the open right halfplane for some $m \geq \operatorname{ind}(A)$ :

$$
A^{\mathrm{D}}=\int_{0}^{\infty} \exp \left(-t A^{m+1}\right) A^{m} d t
$$

It is natural to ask whether we can drop the restriction on the spectrum of $A^{m+1}$. In this note we will establish an integral representation for the Drazin inverse $A^{\mathrm{D}}$ which holds without any restriction on the eigenvalues of $A$.

${ }^{*}$ Received by the Editors on 5 September 2001. Final manuscript accepted for publication on 11 July 2002. Handling Editor: Daniel Hershkowitz.

${ }^{\dagger}$ Facultad de Informática, Universidad Politécnica de Madrid, Campus de Montegancedo, Boadilla del Monte, 28660 Madrid, Spain (nieves@fi.upm.es).

${ }^{\ddagger}$ Department of Mathematics and Statistics, University of Melbourne, VIC 3010, Australia (j.koliha@ms.unimelb.edu.au).

$\S$ Department of Mathematics and Laboratory of Mathematics for Nonlinear Sciences, Fudan University, Shanghai 200433, People's Republic of China (ymwei@fudan.edu.cn). Supported by the National Natural Science Foundation of China (under grant 19901006) and the Doctoral Point Foundation of China. Partial work was done when this author visited Harvard University, supported by the China Scholarship Council. 
2. Integral representation for the Drazin inverse $A^{\mathrm{D}}$. We mention that for the Moore-Penrose inverse $A^{\dagger}$ of a matrix $A \in \mathbb{C}^{n \times n}$ (and more generally of a bounded Hilbert space operator $A$ with closed range) there is a well known integral representation due to Showalter [3],

$$
A^{\dagger}=\int_{0}^{\infty} \exp \left(-t A^{*} A\right) A^{*} d t
$$

generalized recently by Wei and Wu to the weighted Moore-Penrose inverse [4].

Our main result which follows bears a certain resemblance to this representation.

Theorem 2.1. Suppose that $A \in \mathbb{C}^{n \times n}$ and $k=\operatorname{ind}(A)$. Then

$$
A^{\mathrm{D}}=\int_{0}^{\infty} \exp \left[-t A^{k}\left(A^{2 k+1}\right)^{*} A^{k+1}\right] A^{k}\left(A^{2 k+1}\right)^{*} A^{k} d t
$$

Proof. For each matrix $A \in \mathbb{C}^{n \times n}$ there exists a nonsingular matrix $P$ such that

$$
A=P\left[\begin{array}{cc}
C & 0 \\
0 & N
\end{array}\right] P^{-1},
$$

where $C$ is a nonsingular matrix and $N$ is a nilpotent matrix of index $k$; either block $C$ or block $N$ may be empty.

The Drazin inverse of $A$ can be then expressed by

$$
A^{\mathrm{D}}=P\left[\begin{array}{cc}
C^{-1} & 0 \\
0 & 0
\end{array}\right] P^{-1}
$$

We partition the Hermitian matrices $P^{*} P$ and $\left(P^{*} P\right)^{-1}$ into block matrices compatible with the above partitioning of $A$ (and $A^{\mathrm{D}}$ ):

$$
P^{*} P=\left[\begin{array}{ll}
P_{11} & P_{12} \\
P_{12}^{*} & P_{22}
\end{array}\right], \quad\left(P^{*} P\right)^{-1}=\left[\begin{array}{ll}
Q_{11} & Q_{12} \\
Q_{12}^{*} & Q_{22}
\end{array}\right]
$$

Since $P^{*} P$ and $\left(P^{*} P\right)^{-1}$ are positive definite Hermitian matrices, so are the submatrices $P_{11}$ and $Q_{11}$. By a direct computation we obtain

$$
\begin{aligned}
A^{k}\left(A^{2 k+1}\right)^{*} A^{k} & =P\left[\begin{array}{cc}
C^{k} & 0 \\
0 & 0
\end{array}\right]\left(P^{*} P\right)^{-1}\left[\begin{array}{cc}
\left(C^{2 k+1}\right)^{*} & 0 \\
0 & 0
\end{array}\right] P^{*} P\left[\begin{array}{cc}
C^{k} & 0 \\
0 & 0
\end{array}\right] P^{-1} \\
& =P\left[\begin{array}{cc}
C^{k} & 0 \\
0 & 0
\end{array}\right]\left[\begin{array}{ll}
Q_{11} & Q_{12} \\
Q_{12}^{*} & Q_{22}
\end{array}\right]\left[\begin{array}{cc}
\left(C^{2 k+1}\right)^{*} & 0 \\
0 & 0
\end{array}\right]\left[\begin{array}{cc}
P_{11} & P_{12} \\
P_{12}^{*} & P_{22}
\end{array}\right]\left[\begin{array}{cc}
C^{k} & 0 \\
0 & 0
\end{array}\right] P^{-1} \\
& =P\left[\begin{array}{cc}
C^{k} Q_{11}\left(C^{2 k+1}\right)^{*} P_{11} C^{k} & 0 \\
0 & 0
\end{array}\right] P^{-1}
\end{aligned}
$$

Similarly, we get

$$
A^{k}\left(A^{2 k+1}\right)^{*} A^{k+1}=P\left[\begin{array}{cc}
C^{k} Q_{11}\left(C^{2 k+1}\right)^{*} P_{11} C^{k+1} & 0 \\
0 & 0
\end{array}\right] P^{-1} .
$$


Write $\sigma(A)$ for the spectrum of $A$ (that is, the set of all eigenvalues of $A$ ). Then

$$
\begin{aligned}
\sigma\left[C^{k} Q_{11}\left(C^{2 k+1}\right)^{*} P_{11} C^{k+1}\right] & =\sigma\left[Q_{11}\left(C^{2 k+1}\right)^{*} P_{11} C^{2 k+1}\right] \\
& =\sigma\left[Q_{11}^{1 / 2}\left(C^{2 k+1}\right)^{*} P_{11}^{1 / 2} P_{11}^{1 / 2} C^{2 k+1} Q_{11}^{1 / 2}\right] \\
& =\sigma\left[\left(P_{11}^{1 / 2} C^{2 k+1} Q_{11}^{1 / 2}\right)^{*}\left(P_{11}^{1 / 2} C^{2 k+1} Q_{11}^{1 / 2}\right)\right],
\end{aligned}
$$

where the last spectrum is positive being the spectrum of a positive definite Hermitian matrix. Thus

$$
\begin{aligned}
\int_{0}^{\infty} & \exp \left[-t A^{k}\left(A^{2 k+1}\right)^{*} A^{k+1}\right] A^{k}\left(A^{2 k+1}\right)^{*} A^{k} d t \\
& =P\left[\begin{array}{cc}
\int_{0}^{\infty} \exp \left[-t C^{k} Q_{11}\left(C^{2 k+1}\right)^{*} P_{11} C^{k+1}\right] d t & 0 \\
0 & 0
\end{array}\right]\left[\begin{array}{cc}
C^{k} Q_{11}\left(C^{2 k+1}\right)^{*} P_{11} C^{k} & 0 \\
0 & 0
\end{array}\right] P^{-1} \\
& =P\left[\begin{array}{cc}
{\left[C^{k} Q_{11}\left(C^{2 k+1}\right)^{*} P_{11} C^{k+1}\right]^{-1}} & 0 \\
0 & 0
\end{array}\right]\left[\begin{array}{cc}
C^{k} Q_{11}\left(C^{2 k+1}\right)^{*} P_{11} C^{k} & 0 \\
0 & 0
\end{array}\right] P^{-1} \\
& =P\left[\begin{array}{cc}
C^{-1} & 0 \\
0 & 0
\end{array}\right] P^{-1} \\
& =A^{\mathrm{D}}
\end{aligned}
$$

This completes the proof.

\section{REFERENCES}

[1] N. Castro González, J. J. Koliha, and Yimin Wei. On integral representations of the Drazin inverse in Banach algebras. Proceedings of the Edinburgh Mathematical Society, 45:327$331,2002$.

[2] J. J. Koliha and Ivan Straškraba. Power bounded and exponentially bounded matrices. Applications of Mathematics, 44:289-308, 1999.

[3] D. Showalter. Representation and computation of the pseudoinverse. Proceedings of the American Mathematical Society, 18:584-586, 1967.

[4] Yimin Wei and Hebing Wu. The representation and approximation for the weighted MoorePenrose inverse. Applied Mathematics and Computation, 121:17-28, 2001. 